\title{
Modification of the Vipulanandan rheological model with correlation for temperature and electrolyte effect on drilling muds.
}

AFOLABI, R.O., YUSUF, E.O. 


\section{Research Article}

Title: Modification of the Vipulanandan Rheological Model with Correlation for Temperature and Electrolyte Effect on Drilling Muds.

Authors: Richard O. Afolabi ${ }^{1, *}$, Esther O. Yusuf ${ }^{2}$

Affiliations: ${ }^{1}$ Petroleum Engineering Research Group (PERG), School of Engineering, Robert Gordon University, Aberdeen, AB10 7GJ, United Kingdom.

${ }^{2}$ Department of Chemical Engineering, Covenant University, P.M.B 1023, Ota, Ogun State, Nigeria.

*Contact email: $\underline{\text { r.afolabi@rgu.ac.uk }}$ 


\begin{abstract}
Modelling the flow of drilling muds requires the use of existing generic time-independent models with a number of parameters incorporated. While these parameters quantify the uncertainties surrounding the mud rheology, the tuning of each parameter for proper flow description can be challenging and time consuming. As such, the introduction of new parameters in describing additional reservoir conditions associated with drilling muds would only increase the complexity of the computational process. Therefore, the predictive capability of applied rheological models for the different flow regimes and reservoir conditions associated with drilling muds is still limited in scope. Existing procedure using generic rheological models would give a generalized approach to computing the performance of drilling muds when taking into account new factors such as temperature and electrolyte effects. Therefore, there is need to accurately model the rheology of drilling muds taking into account new conditions without necessarily introducing new fitting parameters to a particular model. In this work, a novel approach was employed where the time constant of the modified Vipulanandan model was related to reservoir conditions without introducing a new fitting constants or parameters. The selection of the Vipulanandan model among others was based on the conditions stated by Vipulanandan and Mohammed (2014) and the use of statistical validation tools based on best fit. Moreover, the Vipulanandan model represents the only model which can be modified to have a time constant in it.
\end{abstract}

Keywords: Drilling Mud; Bentonite; Shear Stress; Rheology; Computational Modelling 


\section{Introduction}

Bentonite clays used in the formulation of drilling mud are in powder form with the solid particles conveniently divided into three groups according to [1]:

(1) Colloids, from about 0.005 to 1 micron $(1$ micron $=0.001 \mathrm{~mm})$, which impart viscous and filtration properties;

(2) Silt and barite (sometimes called "inert solids"), 1 to 50 microns, which provide density as just discussed but are otherwise deleterious; and,

(3) Sand, 50 to 420 microns (assuming a 40-mesh screen on the shale shaker), which, apart from bridging large openings in certain very porous formations, is objectionable because of its abrasive qualities.

The description of the rheology of drilling muds is essential for adequate determination of hydraulic conditions such as velocity profile and pressure loss emanating during drilling activities [2]. This is also significant in the estimation of the hole cleaning efficiency of the drilling mud [2 - 7]. Therefore, computational modelling of the velocity profile, pressure loss and hole cleaning efficiency during drilling requires models which can approximate the rheology of the mud. The application of known rheological models in the description of flow behaviour of drilling muds necessitates that its predictive capability correlates with certain conditions. Mathematically, Vipulanandan and Mohammed [8] described these conditions as shown in equations (1) to (4):

$$
\begin{aligned}
& \lim _{\dot{\gamma} \rightarrow 0} \tau=\tau_{0} \\
& \frac{\mathrm{d} \tau}{\mathrm{d} \dot{\gamma}}>0 \\
& \frac{\mathrm{d}^{2} \tau}{\mathrm{d} \dot{\gamma}^{2}}<0
\end{aligned}
$$


$\lim _{\dot{\gamma} \rightarrow \infty} \tau=\tau_{\max }$

Where $\tau_{0}$ is the yield point, $\tau$ is the shear stress and $\dot{\gamma}$ is the shear rate of the drilling mud. Equation (1) simply describes the yield point of the drilling mud. This is the minimum shear stress that must be exceeded for the drilling mud to flow. Furthermore, it is a measure of the pumping ability of the drilling mud and its efficiency in the removal of drilled cuttings under static and dynamic conditions respectively [8 - 13]. In addition, equation (2) indicates that the drilling mud must possess sufficient viscosity in order to keep the weighting materials and drilled cuttings suspended during continuous mud circulation. The absence of sufficient viscosity would result in the cuttings or weighting materials settling out of suspension when mud circulation is stopped $[6,13,14]$. The thixotropic nature of drilling muds is captured in equation (3) where there is a reversible decrease in viscosity with shear rate and increase in viscosity when the shear is removed. Moreover, the maximum shear stress tolerance of the dilling mud is captured in equation (4). The shear stress limit of a drilling mud indicates its erosive capability which is an important function of drilling muds $[8,13]$. Asides the breaking of rocks by the drill bit, the drilling muds must also contribute to this through its erosive potential. However, computational modelling of the flow behaviour of drilling muds is still limited in scope with reliance placed on existing models such as the Bingham plastic model, Herschel-Buckley, Power-Law, Robertson-Stiff, Casson, Sisko, and Prandtl-Eyring. These generic rheological models would give a generalized approach to computing the performance of drilling muds when taking into account new factors such as thermal and salinity effects. Tchameni et al. [15] used a similar generalized approach when investigating the temperature effect (varied over 28 to $180{ }^{\circ} \mathrm{C}$ ) on the rheology of waste vegetable oil biodiesel modified bentonite drilling mud using the Vipulanandan model. It was observed that the Vipulanandan model predicted the shear thinning relationship between the shear stress and shear strain rate of the modified bentonite drilling muds satisfactorily when compared to other models. 
However, the generalized approach meant that the parameter constants of the Vipulanandan model would need to be fitted to the various temperature values under consideration. This would only result in additional fitting parameters in order to describe the flow characteristics of the waste vegetable oil biodiesel modified bentonite drilling mud. Similarly, Fan et al. [16] introduced a mathematical model for the computational modelling of drilling muds under high temperature and high pressure (HPHT) conditions. The approach by the authors introduced new dimensionless parameters to explicitly account for the effect of temperature and pressure. The multi-parameter nature of the developed model generally would give a full description of drilling mud rheological flow. However, while they had better quantify the uncertainties surrounding the rheology of the drilling muds, the manual tuning of each parameter for proper flow description can be challenging and time consuming for simulation purpose. For that reason, the large number of model parameters can be challenging and not easy to estimate via non-linear regression analysis. Currently, the use of existing time independent rheological models in its present form for drilling muds would require a data-driven approach where models are regressed to shear stress-shear rate values [17]. Furthermore, accounting for the effect of new conditions would require the rheological constants of the models to be expressed as a function of new factors of interest (such as temperature or salinity). This means that more fitting parameters would be introduced in a similar way as reported by Afolabi et al. [7], Vipulanandan and Mohammed [8], Fan et al. [16], Mohammed [18] and Afolabi et al. [19]. In this work, a novel approach was presented where the time constant of a selected rheological model is related to reservior conditions without introducing a new dimensionless fitting parameter. The selection of a particular rheological model was based on the conditions stated by Vipulanandan and Mohammed [8] in equations (1) to (4). In addition, the use of statistical validation tools based on best fit were applied in the comparison of the various timeindependent models. Moreover, the Vipulanandan model represents the only model which can 
be modified to have a time constant in it. This approach ensured that the time constant in the Vipulanandan model can be correlated to variation in reservoir conditions such as temperature and salinity (or electrolyte concentrations).

\section{Theory and Modification of the Vipulanandan Model}

Table 1 show some specific rheological models and their predictive capability based on the conditions presented in equations (1) to (4). Based on these conditions, the Vipulanandan model proposed by Vipulanandan and Mohammed [8] which has a limit on the shear stress for a drilling mud was considered among others for this study as shown in equation (5) below

$\tau=\tau_{0}+\frac{\dot{\gamma}}{\mathrm{A}+\mathrm{D} \dot{\gamma}}$

Where $\dot{\gamma}$ is the shear rate $\left(\mathrm{s}^{-1}\right), \tau_{0}$ is the yield stress $(\mathrm{Pa}), \mathrm{A}\left([\mathrm{Pas}]^{-1}\right)$ and $\mathrm{D}\left(\mathrm{Pa}^{-1}\right)$ are model parameters or constants respectively. The ratio of the model constants (D/A) can be taken to represents a time constant which characterizes the transition from Newtonian to shear thinning flow. According to Lohne et al. [20], the clay particles can be considered to spend longer time oriented in the flow direction during tumbling motion thereby resulting in shear thinning. The shear erosive potential of the drilling mud can be predicted by its shear stress limit, $\tau_{\text {lim }}$ according to equation (6).

$\lim _{\gamma \rightarrow \infty} \tau=\tau_{\lim }=\tau_{0}+\frac{1}{D}$

The ratio of the model constants (D/A) represents a time constant denoted $\beta_{1}$ and this can be represented as shown in (7)

$\beta_{1}=\frac{\mathrm{D}}{\mathrm{A}}$

Modifying equation (5) to take into account this time constant yields equation (8) with $\beta_{2}=$ 1/A (Pas). 
$\tau=\tau_{0}+\frac{\beta_{2} \dot{\gamma}}{1+\beta_{2} \dot{\gamma}}$

According to Lohne et al. [20], this time constant can be obtained by considering the characteristic time when Brownian motion is overcome by hydrodynamic forces. Thus, the expression for the time constant can be related to a characteristic time scale of rotational diffussion, $\mathrm{D}_{\text {rot }}$ as shown in (9)

$\beta_{1}=\frac{1}{2 \mathrm{D}_{\mathrm{rot}}}$

This rotational diffussion coefficient, $\mathrm{D}_{\text {rot }}$ can be computed using the Einstein-Smoluchowski relation and approximated to the final expression as shown in (10) below

$\mathrm{D}_{\text {rot }}=\frac{\mathrm{k}_{\mathrm{B}} \mathrm{T}}{f}$

Where $f$ is the friction factor and be expressed as equation (11)

$f=8 \pi \mu_{\mathrm{s}} \mathrm{R}_{\mathrm{h}}^{3}$

Substituting (11) into (10) yields

$D_{\text {rot }}=\frac{k_{B} T}{8 \pi \mu_{s} R_{h}^{3}}$

Where $\mathrm{k}_{\mathrm{B}}$ is the Boltzmann constant, $\mu_{\mathrm{s}}$ is the solvent (or water) viscosity, $\mathrm{T}$ is the temperature and $R_{h}$ is the hydrodynamic radius of a rigid sphere in solution. Substituting (12) into (9) and simplifying yields the final expression in (13)

$\beta_{1}=\beta_{0}\left(\frac{\mu_{\mathrm{s}} \mathrm{R}_{\mathrm{h}}^{3}}{\mathrm{~T}}\right)$

Where $\beta_{0}=4 \pi / \mathrm{k}_{\mathrm{B}}$ with a theoretical value of $9.1018 \times 10^{23} \mathrm{~K} / \mathrm{J}$ and it is considered as a tuning parameter to account for approximations in the assumption of a spherical clay particles and the uncertainty arising from the effects of polydispersity of the bentonite clay particles. In 
order words, the prefactor $\beta_{0}$ was used to account for the variation in the interaction between clay particles. The value of $\beta_{0}$ would also change due to the effects arising from variation in temperature and salinity. The solution procedure used in the predictive modelling of the variation in the interaction between bentonite clay particles is summarized in Figure 1.

\subsection{Adjusting for Temperature Effects}

The time constant, $\beta_{1}$ is an inverse function of temperature since it was related to diffusion of spherical molecules $[20,21]$. Therefore, the time constant in the modified Vipulanandan model from (13) already takes into account temperature effect. However, the viscosity of drilling muds is assumed to be temperature dependent due to the fact the solvent viscosity changes with temperature [20, 21]. According to Brakstad and Rosenkilde [21], the solvent viscosity can be assumed to change with temperature from a reference temperature $T_{0}$ to temperature $T$ according to an empirical Arrhenius type equation shown in (14)

$\mu_{\mathrm{s}}(\mathrm{T})=\mu_{\mathrm{s}}\left(\mathrm{T}_{\mathrm{o}}\right) * \exp \left(\frac{\mathrm{E}_{\mathrm{a}}}{\mathrm{R}_{\mathrm{g}}}\left[\frac{1}{\mathrm{~T}}-\frac{1}{\mathrm{~T}_{\mathrm{o}}}\right]\right)$

Where $E_{a}$ is the activation energy and $R_{g}$ is the ideal gas constant. The viscosity of numerous of liquids or its mixtures obey the Arrhenius behaviour under varying temperatures. This is based on simple thermodynamic considerations where movement of fluids is a local transition of a molecule or a group of molecules from one state to another after overcoming an energy barrier [22].

\subsection{Adjusting for Salinity Effects}

The effect of salinity or electrolytes is included in equation (13) by making the solvent viscosity salinity dependent according the approach of Brakstad and Rosenkilde [21] as shown in equation (15)

$\mu_{\mathrm{s}}=\frac{\mu_{\mathrm{s}, \mathrm{eff}}}{\mathrm{k}_{\mu}}$ 
$\mu_{\mathrm{s}, \mathrm{eff}}=\mu_{\mathrm{s}} \mathrm{k}_{\mu}$

Where $\mu_{\mathrm{s}, \mathrm{eff}}$ represents the corrected solvent viscosity for salinity, $\mathrm{k}_{\mu}$ is a salinity correction factor taking into account as well the effect of temperature and can be represented by (17) as obtained by Brakstad and Rosenkilde [21].

$\mathrm{k}_{\mu}=1+\mathrm{A}_{1} \mathrm{C}_{\mathrm{s}}+\mathrm{A}_{2} \mathrm{C}_{\mathrm{s}}^{2}$

Where $C_{s}$ is the total sum of salts in solution in $g / L$. The constants $A_{1}$ and $A_{2}$ in equation (17) are temperature dependent constants given by

$$
\begin{aligned}
& \mathrm{A}_{1}=\left(1.48 \times 10^{-3} \frac{\mathrm{g}}{\mathrm{L}}\right)+\left(1.50 \times 10^{-5} \frac{\mathrm{g}}{\mathrm{L}^{\circ} \mathrm{C}}\right) \mathrm{T}-\left(3.93 \times 10^{-8} \frac{\mathrm{g}}{\mathrm{L}^{\circ} \mathrm{C}^{2}}\right) \mathrm{T}^{2} \\
& \mathrm{~A}_{2}=\left(1.07 \times 10^{-5} \frac{\mathrm{g}}{\mathrm{L}}\right)-\left(8.50 \times 10^{-8} \frac{\mathrm{g}}{\mathrm{L}^{\circ} \mathrm{C}}\right) \mathrm{T}+\left(2.23 \times 10^{-10} \frac{\mathrm{g}}{\mathrm{L}^{\circ} \mathrm{C}^{2}}\right) \mathrm{T}^{2}
\end{aligned}
$$

\section{Material and Methods}

\subsection{Materials}

The bentonite clay was acquired from a local supplier in Nigeria. The clay storage condition in the laboratory was between 27 to $30{ }^{\circ} \mathrm{C}$. The mineralogical analysis was carried out using the PAN Analytical X-Pert Pro diffractometer operating at $30 \mathrm{kV}$ and $40 \mathrm{~mA}$. The mineral composition is contained in Table 2. Investigation of the elemental composition of the bentonite clay was done using Energy Dispersive X-Ray (EDX) spectroscopy. This was carried out using the Phenom ${ }^{\circledR}$ ProX desktop Energy Dispersive X-Ray (EDX) machine as reported in Table 3. Particles size distribution of the bentonite clay was measured using a laser scattering particle size analyzer $\left(\right.$ Shimadzu $^{\circledR}$ Particle Size Analyzer). The average particle size for the bentonite clay is $4 \mu \mathrm{m}$ (Figure 2). The electrolytes considered for this study were Sodium Chloride $(\mathrm{NaCl}$; ACS reagent, $\geq 99.0 \%)$ and Calcium Chloride $\left(\mathrm{CaCl}_{2} \cdot 7 \mathrm{H}_{2} \mathrm{O}\right.$; ACS reagent, $\geq$ 
$99 \%)$ which are manufactured by Sigma Aldrich. The materials were all purchased from Equilab Solutions in Nigeria.

\subsection{Formulation of Drilling Mud}

Synthetic brine was formulated using varying concentration of $\mathrm{NaCl}$ (for salinity) and $\mathrm{CaCl}_{2}$ (for hardness) in $350 \mathrm{~mL}$ of deionized water. The brine was prepared containing both $\mathrm{NaCl}$ and $\mathrm{CaCl}_{2}$ in the ratios 10 to 1 respectively. The composition of the brine was:

- $\mathrm{NaCl}: 0.1,0.3,1.0$, and $10 \%$

- $\mathrm{CaCl}_{2}: 0.01,0.03,0.1$, and $1 \%$

A Hamilton beach mixer was used to continuously stir the brine solutions until the formation of a homogenous solution. The brine solutions act as the medium for the dissolution of bentonite clays thereby giving rise to bentonite muds. The stirring speed of the mixer was set to $11000-$ RPM. The drilling muds was prepared by adding $6.3 \mathrm{wt} . \%, 13 \mathrm{wt} \% \%$ and $15 \mathrm{wt} . \%$ of bentonite clay to different concentrations of brine solutions followed by mixing for 20 minutes.

\subsection{Rheological Measurement}

The flow characteristics of the drilling mud was evaluated using an OFITE Model 800 (8Speed) Viscometer that is manufactured by OFI Testing Equipment, Inc. The rheological behaviour was obtained by measuring the shear stress at different shear rates. The shear rates were simply altered with a speed regulator, which was done to sustain a continuous shear rate under changing shear conditions and input power. The values for shear stress were shown on an illuminated enlarged dial for easy reading. The dial readings (DR) from the viscometer were taken at equilibrium values. The eight accurately controlled test speeds of the viscometer (shear rates in RPM) are 3 (Gel), 6, 30, 60, 100, 200, 300, and 600. 


\section{Results and Discussion}

\subsection{Comparison between Vipulanandan and other Rheological Models}

Comparison between the Vipulanandan model and the 3 most common rheological models (Power, Bingham and Herschel Buckley) employed in the oil and gas industry is shown in Figure 3. The value of the coefficient of determination, $R^{2}$, for the Power law model for a base case without electrolytes was 0.962 for $6.3 \mathrm{wt} . \%$ bentonite mud with the root mean square error (RMSE) being $1.8654 \mathrm{~Pa}$. For the Bingham Plastic model, the $R^{2}$ value of 0.991 and RMSE value of $1.039 \mathrm{~Pa}$ was obtained for $6.3 \mathrm{wt} . \%$ bentonite mud. In the case of the Herschel Buckley model, the base case mud of $6.3 \mathrm{wt} . \%$, bentonite content was modelled with, $R^{2}$, value of 0.999 and RMSE value of 0.433 Pa respectively. The Vipulanandan model with shear stress limit prediction was fitted with a $R^{2}$ value of 0.999 and RMSE value of $0.377 \mathrm{~Pa}$ for $6.3 \mathrm{wt} . \%$ bentonite mud. The Vipulanandan and Herschel Buckley models showed comparable values for $R^{2}$. However, the RMSE value for the Vipulanandan model was lower $(0.377 \mathrm{~Pa})$ compared to the Herschel Buckley model (0.433). This indicates better fitting of the Vipulanandan model to the rheological data. Further comparison between the Vipulanandan and Herschel Buckley models was done using the confidence and prediction intervals. Figure 4 show the $95 \%$ confidence interval for the Vipulanandan and Herschel Buckley models. The tapered confidence interval connected with the models is suggestive of their accuracy in predicting the shear stress for a definite set of the predictor variable which is the shear rate. Furthermore, in accessing the applicability of the Vipulanandan model, the uncertainty of predicting the value of a single future observation or a fixed number of multiple future observations based on the distribution of previous observations was evaluated. This was done using the prediction interval, which is the range that is likely to contain a single future response for a selected combination of variable settings. Figure 5 show the $95 \%$ prediction interval for the Vipulanandan and Herschel Buckley models. There is $95 \%$ probability that future observation 
will be contained within the prediction interval. Therefore, the Vipulanandan model shows comparable fitting attributes with the Herschel Buckley rheological model employed in the oil and gas industry. However, the capability of the Vipulanandan model is extended above the Herschel Buckley model due to its prediction of the shear stress limit.

\subsection{Predicting the Effect of Bentonite Content on Mud Rheology}

Figure 6 show the fitted Vipulanandan model to bentonite mud containing varying clay concentration $(6.3,13$ and 15 wt.\%). The model show good fitting to the rheological data irrespective of the bentonite clay content. The model time constant, $\beta_{1}$ and tuning parameter, $\beta_{0}$ for the varied bentonite content is shown in Table 4 . The tuning parameter increased with bentonite content up to $13 \mathrm{wt} . \%$ after which it decreased. The tuning parameter is assumed to account for the dispersion of clay particles arising from hydration in water. The increase in clay content would increase the amount of clay particles susceptible to hydration and swelling in water. However, the randomness of particle dispersion in solution may explain the non-uniform trend of the tuning parameter as shown in Figure 7 and subsequently in Figure 8 in the next section. It is obvious that allowing the tuning parameter vary rather than have it as a constant accounted for additional characteristics associated with the dispersion of the clay particles.

\subsection{Predicting the Effect of Electrolyte Concentration on Mud Rheology}

The bentonite clay particles consist mainly of the mineral montmorillonite, which are planar and have two kinds of surface charges. On the faces, a negative surface charge is formed from the substitutions within the interior of the crystal lattice. At the edge of the clay particles, a positive surface charge is formed. This special charge distribution gives way to different modes of clay particle association. Table 5 contains the values of the Vipulanandan model constants based on equation (8) and (13) respectively for $13 \mathrm{wt} . \%$ bentonite mud at $25{ }^{0} \mathrm{C}$ with the corresponding plastic viscosity (PV) and apparent viscosity (AV). Figure 8 shows the variation of the predicted tuning parameter $\beta_{0}$ using the Vipulanandan model and the corresponding 
change in the rheological properties of the bentonite mud. The reduction in the value of $\beta_{0}$ is indicative of the variation in the state of the clay particles arising from the addition of electrolytes. There was an initial reduction of approximately $37 \%$ in the value of $\beta_{0}$ followed by an increase and a slight decrease afterwards. This can be attributed to the strong initial destabilizing effect of the cations. The subsequent increase is due to the remaining and existing interaction between clay particles which tries to maintain the initial state of the particle suspension. The calcium ions which is divalent can effectively associate with two negative charges compared to the sodium ions and this will provide strong effect on clay platelet sheets thereby binding them together. Similarly, as the concentration of cations is increased, the degree of hydration is further reduced and agglomeration of bentonite clay particles is increased. This is caused by the presence of additional cations $\left(\mathrm{Na}^{+}\right.$and $\left.\mathrm{Ca}^{2+}\right)$ in solution providing more interaction with the surface of the clay platelet. This further destabilizes the equilibrium interaction between clay particles leading to the creation of new thermodynamic states. More so, this causes the ion density in the water layer bound by the clay surface and the exchange cations to be reduced resulting in the reduction in the viscosity of the bentonite mud and in the value of $\beta_{0}$. This effectively reduce the clay particle distance and explains the poor rheology of the electrolyte treated mud. The variation in the value of $\beta_{0}$ can as well be viewed to account the change in the equilibrium state arising from the distortion and reformation of clay particle interaction. The non-uniformity of the plot of $\beta_{0}$ with electrolyte concentration as shown in Figure 8 can also be interpreted in terms of the randomness of the clay particles in solution before the formation of new thermodynamic states. However, more research would be needed in this area to further understand the phenomenon associated with the tuning parameter $\beta_{0}$. This would further enhance the applicability of the Vipulanandan model to account for not only the effect of electrolytes on the rheology of bentonite muds but also the thermodynamic state of the clay particles. 


\subsection{Predicting the Effect of Temperature on Mud Rheology}

The model time constant, $\beta_{1}$ and tuning parameter, $\beta_{0}$ for the effect of temperature on mud rheology is shown in Table 6 . The tuning parameter initially decreased with temperature up to $30{ }^{0} \mathrm{C}$ after which it increased. The increase in temperature would increase the thermal or kinetic energy of clay particles. However, this would impact on the viscosity of the bentonite mud as shown by a reduction in the plastic viscosity in Figure 9. Similarly, as the thermal energy is increased beyond the hydration energy of the clay particles, the solvation and swelling of the energetic clay particles is reduced. This causes the water layer bound by the clay surface to be reduced resulting in the reduction in the viscosity of the bentonite mud. Therefore, the dispersion of the clay particles is reduced resulting in a reduction in the clay particle distance. This is evident from the graphical trend of the tuning parameter of the Vipulanandan model as shown in Figure 9. The increase in the tuning parameter show the increased particle to particle interaction arising from increased electrostatic repulsion between exchangeable cations which exists on the surface of the clay. The reduction in the solvation of the clay particles means that the exchangeable cations on the surface cannot interact with water molecules and would be exposed for electrostatic repulsion. However, more experimental studies are required to understand how this tuning parameter relates with reservoir temperature conditions. With typical reservoir temperature conditions between $85-90{ }^{\circ} \mathrm{C}$, clay particle dispersion would be affected and this would be reflected in the changes associated with the tuning parameter. Similarly, reservoir salinity conditions could be as high as $30,000 \mathrm{ppm}$ and this would impact on clay particle interaction. Experimentally, more study is needed to correlate this effect to the tuning parameter of the modified Vipulanandan model.

\subsection{Predicting the Shear Stress Limit and Experimental Validation.}

The shear erosive potential of the bentonite mud using equation (8) can be predicted according to equation (20). 
$\lim _{\gamma \rightarrow \infty} \tau=\tau_{\lim }=\left[\tau_{0}+\frac{\beta_{2}}{\beta_{1}}\right]$

Where $\tau_{\lim }(\mathrm{Pa})$ is the shear stress limit, which is a measure of the extent of shear stress tolerance of the bentonite mud. The shear stress limit was predicted using equation (20) for bentonite mud containing 6,9 and $11 \mathrm{wt} . \%$ bentonite dispersed. The values for the shear stress limit predicted were 15.32, 33.71 and $63.8 \mathrm{~Pa}$ for 6,9 and $11 \mathrm{wt} . \%$ bentonite respectively. The experimental approach used in validating the predicted shear stress limit values was the shear loading method. In this approach, a given shear rate $\left(1022 \mathrm{~s}^{-1}\right)$, which corresponds to the structural breakdown of the bentonite mud was applied using the OFITE model 800 viscometer for a period of 15 minutes. After a steady value for the dial reading (DR) was obtained, the applied shear rate was reduced to zero and the gelling or recovery (structural recovery) was noted for the same time period as the structural breakdown. The DR after the recovery was then noted and the process was repeated until the DR after structural breakdown is constant. At this point, the bentonite mud has yielded and the DR was noted and compared with the prediction as derived from equation (20). The values estimated from the experiment compared to the predictions of the new model are summarized in Table 7. The plots of the shear stress versus time showing the shear loading-shear recovery of the bentonite mud is shown in Figure 10. The open markers refers to the point where the shear stress values remain constant and approximates the predicted values for shear stress limit by equation (20). Extending shearing time beyond what was applied in this study would result in a decrease in the values of the DR beyond the shear stress limit. This is indicative of the structural degradation of the bentonite mud and would result in an irreversible loss in viscosity.

\section{Conclusion and Recommendation}

This study was carried out to develop a new predictive approach to the modelling of the rheological behaviour of drilling muds under certain reservoir conditions. Statistical 
comparison between the Vipulanandan model and the three commonest time-independent models used in the oil and gas industry was done. The Vipulanandan model showed similar comparison with the Herschel Buckley with the two models describing mud rheology more effectively than the rest. However, among the various time-independent models considered, the Vipulanandan model has a robust predictive capability which is consistent with the conditions of Vipulanandan and Mohammed (2014). This robustness was further demonstrated in this work by the modification of the Vipulanandan model in correlating the rheology of drilling muds under certain reservoir conditions. A key advantage of this approach is that the time constant of the modified Vipulanandan model was correlated to reservior conditions such as salinity and temperature without introducing a new fitting constants or parameters. Therefore, the introduction and fitting of new parameters via non-linear regression analysis in describing additional reservoir conditions associated with drilling muds was not required. Rather, the existing time constant parameter of the modified Vipulanandan model was related to the Einstein-Smoluchowski expression to account for the effect of reservoir conditions (temperature and salinity effects). This significantly reduced the complexity of the computational process especially with the use of non-linear regression analysis. The significance of this is the introduction of a tuning parameter which in this work was related to the response of clay particle interaction when such muds are subjected to these conditions of salinity and temperature. However, more studies are still required in the understanding of this tuning parameter and this can be studied through experimental characterization of the particle dispersion state with changes in temperature, salinity or particle concentration. More importantly is the understanding of the trend and shape with respect to the actual conditions in a reservoir with temperature and salinity conditions as high as $85{ }^{\circ} \mathrm{C}$ and $30,000 \mathrm{ppm}$ respectively. This may effectively be linked to the change in the structural state of the drilling mud under these conditions. 


\section{Nomenclature}

Abbreviations

DR Dial Readings

RPM Revolutions per Minutes

PV Plastic Viscosity

YP Yield Point

AV Apparent Viscosity

Symbols

$\begin{array}{ll}\tau_{0} & \text { Yield Point, Pa } \\ \tau & \text { Shear Stress, Pa } \\ \dot{\gamma} & \text { Shear Rate, } \mathbf{s}^{-\mathbf{1}} \\ \tau_{\infty} & \text { Shear Stress measured at high Shear Rates, Pa } \\ \mathrm{k}_{\mathrm{B}} & \text { Boltzmann Constant, } \mathbf{J} / \mathbf{K} \\ \beta_{0} & \text { Tuning Parameter in the Modified Vipulanandan Model, K/J } \\ \beta_{1} & \text { Time Costant in the Modified Vipulanandan Model, } \mathbf{s} \\ \beta_{2} & \text { Parameter Constant in the Modified Vipulanandan Model, Pas } \\ \mathrm{C}_{\mathrm{b}} & \text { Bentonite Content, wt. } \% \\ \mathrm{C}_{\mathrm{s}} & \text { Total Sum of Salts in Solution, } \mathbf{g} / \mathbf{L} \\ \mathrm{D}_{\mathrm{rot}} & \text { Rotational Diffusion Coefficient } \\ \mathrm{E}_{\mathrm{a}} & \text { Activation Energy, } \\ \tau_{\mathrm{lim}} & \text { Gear } / \mathbf{m o l} \\ \mathrm{R}_{\mathrm{g}} & \text { Gas Constant, } \mathbf{J} / \mathbf{m o l} . \mathbf{K} \\ f & \end{array}$


$\mathrm{A}_{2} \quad$ Temperature Dependent Constant in the Salinity Model, $\mathbf{g} / \mathbf{L}$

D Parameter Constant in Vipulanandan Model, $\mathbf{P a}^{\mathbf{- 1}}$

K Consistency Index, $\left[(\mathbf{P a}) \mathbf{s}^{\mathbf{n}}\right]$

$\mathrm{k}_{\mu} \quad$ Salinity Correction Factor for Solvent Viscosity

n Flow Index

$\mu_{\mathrm{p}} \quad$ Plastic Viscosity, Pas

$\mu_{\mathrm{s}} \quad$ Solvent Viscosity, Pas

$\mu_{\mathrm{s}, \mathrm{eff}} \quad$ Corrected Solvent Viscosity, Pas

$\mathrm{a}_{\mathrm{s}}, \mathrm{b}_{\mathrm{s}}$ and $\mathrm{c}_{\mathrm{s}}$ Parameter Constants in Sisko Model,

$\mathrm{R}_{\mathrm{h}} \quad$ Hydrodynamic Radius of a Rigid Sphere, $\mathbf{n m}$

$\gamma_{\mathrm{o}} \quad$ Parameter Constant in Robertson-Stiff \& Modified Robertson-Stiff Model, $\mathbf{s}^{\mathbf{- 1}}$

$A_{t}$ and $B_{p} \quad$ Parameter Constants in Prandtl-Eyring Model.

$\mathrm{T}_{\mathrm{o}} \quad$ Reference Temperature, ${ }^{\circ} \mathrm{C}$

T $\quad$ Temperature, ${ }^{\circ} \mathbf{C}$

\section{Acknowledgement}

The authors would like to appreciate the management of Covenant University for providing an enabling environment to carry out this research.

\section{Funding}

This research did not receive any specific grant from funding agencies in the public, commercial, or not-for-profit sectors.

\section{Conflict of Interest}

The authors have no potential conflict of interest to declare regarding the publication of this article. 


\section{References}

[1] H. C. Darley, G. R. Gray, Composition and Properties of Drilling and Completion Fluids. $5^{\text {th }}$ Ed, Gulf Professional Publishing, 1988.

[2] E. A. Toorman, Modelling the Thixotropic Behaviour of Dense Cohesive Sediment Suspensions. Rheologica Acta, 36 (1997), 56-65.

[3] J. Abdo, M. Danish Haneef, Nano-Enhanced Drilling Fluids: Pioneering Approach to Overcome Uncompromising Drilling Problems. Journal of Energy Resources Technology, 134 (2012), 1-6.

[4] K. P. Hoelscher, G. Stefano, M. Riley, \& S. Young, Application of Nanotechnology in Drilling Fluids. SPE International Oilfield Nanotechnology Conference. Society of Petroleum Engineers. Noordwijk, Holland (2012).

[5] C. M. Jung, R. Zhang, M. Chenevert, M. Sharma, High Performance Water-Based Mud using Nanoparticles for Shale Reservoirs. In: Proc. Unconventional Resources Technology Conference. Society of Petroleum Engineers. Denver, United States (2013).

[6] A. R. Ismail, A. Aftab, Z. H. Ibupoto, N. Zolkifile, The Novel Approach for the Enhancement of Rheological Properties of Water-Based Drilling Fluids by using MultiWalled Carbon Nanotube, Nanosilica and Glass Beads. Journal of Petroleum Science and Engineering, 139 (2016), 264-275.

[7] R. O. Afolabi, O. D. Orodu, V. E. Efeovbokhan, Properties and application of Nigerian bentonite clay deposits for drilling mud formulation: Recent advances and future prospects. Applied Clay Science, 143 (2017a), 39-49.

[8] C. Vipulanandan, A. S. Mohammed, Hyperbolic Rheological Model with Shear Stress Limit for Acrylamide Polymer Modified Bentonite Drilling Muds. Journal of Petroleum Science and Engineering, 122 (2014), 38-47.

[9] V. C. Kelessidis, R. Maglione, Yield Stress of Water-Bentonite Dispersions. Colliods and Surfaces A: Physiochemical and Engineering Aspects, 318 (2008), 217-226.

[10] B. Abu-Jdayil, Rheology of Sodium and Calcium Bentonite-Water Dispersions: Effect of Electrolytes and Aging Time. International Journal of Mineral Processing, 98 (2011), 208-213. 
[11] E. J. Lee, C. Stefanus, Y. K. Leong, Structural Recovery Behaviour of Kaolin, Bentonite and K-Montmorillonite Slurries. Powder Technology, 223 (2012), 105-109.

[12] J. Yoon, J. El-Mohtar, Dynamic Rheological Properties of Sodium Pyrophosphate Modified Bentonite Suspensions for Liquefaction Mitigation. Clays Clay Minerals, 61 (2013), 319-327.

[13] R. O. Afolabi, O. D. Orodu, V. E. Efeovbokhan, O. J. Rotimi, Optimizing the rheological properties of silica nano-modified bentonite mud using overlaid contour plot and estimation of maximum or upper shear stress limit. Cogent Engineering, 4 (2017b), 118.

[14] R. O. Afolabi, E. O. Yusuf, Nanotechnology and the Global Energy Demand: Challenges and Prospects for a Paradigm Shift in the Oil and Gas Industry. Journal of Petroleum Exploration and Production Technology, 9 (2019a), 1423-1441.

[15] A. P. Tchameni, L. Zhao, J. X. Ribeiro, T. Li, Evaluating the thermal effect on the rheological properties of waste vegetable oil biodiesel modified bentonite drilling muds using Vipulanandan model. HTHP Journal, 48 (2019), 207-232

[16] H. Fan, H. Zhou, X. Meng, J. Gao, G. Wang, Accurate Prediction Model for Rheological Properties of Drilling Fluids at High Temperature and High Pressure Conditions. In: Proc. SPE/IATMI Asia Pacific Oil \& Gas Conference and Exhibition. Society of Petroleum Engineers. Bali, Indonesia (2015).

[17] R. O. Afolabi, T. F. Ogunkunle, O. A. Olabode, E. O. Yusuf, Dataset on the beneficiation of a Nigerian bentonite clay mineral for drilling mud formulation. Data in Brief, 20 (2018), 234-241.

[18] A. S. Mohammed, Vipulanandan Model for the Rheological Properties with Ultimate Shear Stress of Oil Well Cement Modified with Nanoclay. Egyptian Journal of Petroleum, 27 (2018), 335-347.

[19] R. O. Afolabi, E. O. Yusuf, Predictive Analytics for the Vipulanandan Rheological Model and its Correlative Effect for Nanoparticle Modification of Drilling Mud. Journal of Petroleum Science and Engineering, 183 (2019b), 106377. 
[20] A. Lohne, O. Nodland, A. Stavland, A. Hiorth, A Model for Non-Newtonian Flow in Porous Media at Diffrent Flow Regimes. Computational Geosciences, 21 (2017), 12891312.

[21] K. Brakstad, C. Rosenkilde, Modelling Viscosity and Mechanical Degradation of Polyacrylamide Solutions in Porous Media, In: Proc. SPE Improved Oil Recovery Conference. Society of Petroleum Engineers, Oklahoma, United States (2016)

[22] T. Budtova, P. Navard, Viscosity-Temperature Dependence and Activation Energy of Cellulose Solutions. Nordic Pulp and Paper Research Journal, 30 (2015), 99-104. 